\title{
COMMENT ON "THE DISTANCE TO THE ORIGIN OF A CERTAIN POINT SET IN En"
}

J. G. WENDEL

In a recent paper [1] Karush and Wolfsohn have considered (in slightly different notation) the problem of finding

$$
M=\min \sum_{i=0}^{m} a_{i}^{2}
$$

Subject to the conditions

$$
\sum_{i=0}^{m} a_{i}=1, \quad \sum_{i=0}^{m} i^{k} a_{i}=0 \quad \text { for } k=1,2, \cdots, r
$$

where $r$ is a fixed integer between 0 and $m-1$ inclusive. After somewhat lengthy calculations they obtain the result

$$
M=1-\frac{(m !)^{2}}{(m+r+1) !(m-r-1) !} .
$$

The purpose of this note is to obtain the result by simpler, more geometrical considerations; in particular we employ the theory, treated for example in $[2, \S 72$ et seq. ], of polynomials orthogonal over a finite set.

Let $P_{k, m}(x) \equiv P_{k}(x)$ be the orthogonal polynomials of degrees $k=0,1, \cdots, m$ on the set $x=0,1, \cdots, m$ having unit constant terms; thus $\sum_{k=0}^{m} P_{k}(x) P_{j}(x)=0$ if $k \neq j$, or, in vector notation, $\left(P_{k}, P_{j}\right)=0$ if $k \neq j$, where $P_{k}$ denotes the vector with components $P_{k}(x), x=0,1, \cdots, m$.

Let $Q_{k}$ be the corresponding unit vectors, $Q_{k}=P_{k} /\left\|P_{k}\right\|$. The squares of the normalizing constants can be written (cf. [2, p. 268, eq. (9) ])

$$
\frac{1}{\left\|P_{k}\right\|^{2}}=\frac{2 k+1}{m+k+1} \frac{(m !)^{2}}{(m+k) !(m-k) !} .
$$

Since $P_{k}(0)=1$ we have

$$
Q_{k}(0)^{2}=\frac{2 k+1}{m+k+1} \frac{(m !)^{2}}{(m+k) !(m-k) !} .
$$

Let $a=\left(a_{0}, a_{1}, \cdots, a_{m}\right)$ be a vector satisfying (1). The inner product of $a$ with any polynomial $Q$ of degree $\leqq r$ is clearly given by

Received by the editors May 18, 1955. 


$$
(a, Q)=Q(0),
$$

and in particular, for the orthonormal polynomials $Q_{k}$,

$$
\left(a, Q_{k}\right)=Q_{k}(0), \quad k=0,1, \cdots, r .
$$

Now the vectors $Q_{k}, k=0,1, \cdots, m$, span $E^{m+1}$ and are orthonormal. Hence any vector $a \in E^{m+1}$ may be written

$$
a=\sum_{k=0}^{m} \alpha_{k} Q_{k}, \quad \quad \alpha_{k}=\left(\alpha, Q_{k}\right)
$$

with

$$
\|a\|^{2}=\sum_{i=0}^{m} a_{i}^{2}=\sum_{k=0}^{m} \alpha_{k}^{2} .
$$

Then the solution to the minimum problem is obtained by choosing

$$
\begin{array}{lr}
\alpha_{k}=Q_{k}(0) & \text { for } k=0,1, \cdots, r, \\
\alpha_{k}=0 & \text { for } k=r+1, \cdots, m .
\end{array}
$$

Hence

$$
\begin{aligned}
M & =\sum_{k=0}^{r} Q_{k}(0)^{2} \\
& =\sum_{k=0}^{r} \frac{2 k+1}{m+k+1} \frac{(m !)^{2}}{(m+k) !(m-k) !} \\
& =\sum_{k=0}^{r} \frac{(m+k+1)-(m-k)}{m+k+1} \frac{(m !)^{2}}{(m+k) !(m-k) !} \\
& =\sum_{k=0}^{r} \frac{(m !)^{2}}{(m+k) !(m-k) !}-\sum_{k=0}^{r} \frac{(m !)^{2}}{(m+k+1) !(m-k-1) !} \\
& =\left(\sum_{k=0}^{r}-\sum_{k=1}^{r+1}\right)\left(\frac{(m !)^{2}}{(m+k) !(m-k) !}\right) \\
& =1-\frac{(m !)^{2}}{(m+r+1) !(m-r-1) !}
\end{aligned}
$$

in agreement with (2).

\section{REFERENCES}

1. W. Karush and N. Z. Wolfsohn, The distance to the origin of a certain point set in $E^{n}$, Proc. Amer. Math. Soc. vol. 6 (1955) pp. 323-332.

2. W. E. Milne, Numerical calculus, Princeton, 1949.

Louisiana State University 